\title{
The Study of Rhabdomyolysis in the Elderly: An Epidemiological Study and Single Center Experience
}

\author{
Supakanya Wongrakpanich*, Christos Kallis, Prithiv Prasad, Janani Rangaswami, Andrew \\ Rosenzweig
}

Department of Medicine, Einstein Medical Center, Philadelphia, Pennsylvania, USA.

[Received January 18, 2017; Revised March 1, 2017; Accepted March 4, 2017]

\begin{abstract}
Rhabdomyolysis is a syndrome caused by injury to skeletal muscle. There is limited data of rhabdomyolysis in the elderly. The objective of this study is to investigate demographic data, etiologies, laboratory values, prognostic factors, and mortality of rhabdomyolysis in the geriatric population. A 4-years retrospective chart review study was conducted. Our inclusion criteria were age above 65 years and creatinine kinase level excess five times of normal upper limit. Among 167 patients, $47.3 \%$ were male. The median age at diagnosis was 80.11 (66-101) years. The duration of follow up in the study ranged from 0 to 48 months. Fall (with or without immobilization) was the most frequent cause of rhabdomyolysis in $56.9 \%$. The mean baseline glomerular filtration rate (GFR), GFR at diagnosis, and peak decline in GFR was 76.94, 48.96, and $54.41 \mathrm{cc} / \mathrm{min}$ respectively. The mean CK at diagnosis and peak CK was 5097.22 and 6320.07 . There were 45 deaths (21\%) over the span of 4 years. Multivariate analysis demonstrated that number of medications pre-admission (Meds No.), peak decline in GFR, and acute kidney injury (AKI) are independent predictors for overall survival for rhabdomyolysis in the elderly. To our knowledge, this is the first epidemiological study of rhabdomyolysis in the elderly. Falls (with and without immobilization) were the most common etiology. Meds No. (>8), peak decline in GFR $(<30 \mathrm{cc} / \mathrm{min})$, and evidence of AKI are associated with shorter overall survival and can serve as potential independent prognostic markers for rhabdomyolysis in elderly patients.
\end{abstract}

Key words: rhabdomyolysis, elderly, immobilization, fall, polypharmarcy

Rhabdomyolysis is a clinical condition caused by injury to skeletal muscle causing leakage of muscle-cell components into body circulation. The common clinical presentations are muscle pain and/or weakness, dark colored urine, and elevation of creatinine kinase (CK), usually more than five times of normal upper limit $[1,2]$. There are multiple known causes of rhabdomyolysis in adults such as strenuous exercise, heat stroke, seizure, delirium tremens, hypokalemia, hypophosphatemia, enzyme deficiencies, trauma, burns, heat stroke myopathy, immobility, hyponatremia, hypernatremia, arterial emboli, endocrinopathies, prolonged immobilization, shock, infections, and medications [3-7].

Vigorous hydration is considered the hallmark for the treatment of this condition. The main purpose is to promote adequate diuresis, correct hypovolemia, and dilute the muscle toxic metabolites from muscle destruction [8-10]. The prognosis of rhabdomyolysis is considered good with overall survival estimated to be around $80 \%$ [11], but varies depend on underlying etiologies and complications [3, 8]. Complications of rhabdomyolysis include electrolyte imbalances,

*Correspondence should be addressed to: Dr. Supakanya Wongrakpanich, Department of Medicine, Einstein Medical Center, Philadelphia, Pennsylvania, USA. Email address: Supakanya.w@gmail.com

Copyright: () 2017 Wongrakpanich S et al. This is an open-access article distributed under the terms of the Creative Commons Attribution License, which permits unrestricted use, distribution, and reproduction in any medium, provided the original author and source are credited. 
arrhythmias, acute kidney injury (AKI), acidosis, compartment syndrome, and disseminated intravascular coagulation [2]. Approximately $30-50 \%$ of patient with rhabdomyolysis will develop AKI [9, 12].

Since Grossman et al. published the early epidemiological data for rhabdomyolysis in 1974 [13], there have been multiple studies regarding rhabdomyolysis in adults[14-18].In 2013, McMahon et al. developed a risk prediction score for mortality in adult rhabdomyolysis patients with age older than 18 years old. They found that age, female sex, cause of rhabdomyolysis, serum creatinine, $\mathrm{CK}$, phosphate, calcium, and bicarbonate are independent prognostic factors of rhabdomyolysis [19].

In the elderly patients, rhabdomyolysis is a common problem, however, there is no dedicated study focusing on the epidemiology and prognosis. The objective of this study was to investigate demographic data, comorbidities, etiologies, incidence of acute kidney injury, laboratory values, prognostic factors, and mortality of rhabdomyolysis in the geriatric population.

\section{MATERIALS AND METHOD}

\section{Subjects}

All elderly patients (age above 65 at the time of diagnosis) who had been diagnosed with rhabdomyolysis (defined by serum creatinine kinase (CK) level excess five times of normal upper limit [1,20]) and visited the Albert Einstein Medical Center as outpatients or inpatients between October 2012 and October 2016 were included in this study. All subjects with preexisting myositis, myopathy, muscular dystrophy, or and CK elevation considered due to acute myocardial infarction were excluded.

One hundred and seventy-one patients fulfilled the inclusion criteria. After 4 patients were excluded, data from 167 patients were analyzed.

\section{Study Design}

We conducted a 4-year retrospective chart review to obtain the following data: age at diagnosis, gender, race, body mass index (BMI), follow-up duration, comorbidities (Hypertension (HTN), diabetes mellitus $(\mathrm{DM})$, congestive heart failure (CHF), coronary artery disease (CAD), Parkinson's disease, dementia, ambulatory dysfunction, cerebrovascular accident (CVA), and chronic kidney disease (CKD)), number of medications pre-admission (Med No.), etiology of rhabdomyolysis, laboratory values, cause of death, and date of death. Overall survival (OS) was defined as the period between the date of diagnosis and the date of death from any cause.
Data was analyzed using SPSS statistics version 17.0. Demographic and descriptive characteristics were summarized using descriptive statistics. Pearson correlation was used to calculate the relationship between peak CK, peak Cr or peak decline in GFR. Univariate and multivariate analyses using Cox proportional hazards regression models were performed to evaluate the independent prognostic factors for rhabdomyolysis in the elderly. A two-sided P-value less than 0.05 was considered to be statistically significant. The study was approved by the Institutional Review Board (IRB) of Philadelphia.

\section{RESULTS}

\section{Patient Characteristics}

Among 167 elderly patients with rhabdomyolysis, 79 (47.3\%) patients were male and 88 (52.7\%) patients were female. African American was the majority race in this study $(73.7 \%)$, followed by Caucasian $(19.8 \%)$. The median age at diagnosis was 80.11 years, with an age range of 66 to 101 years. The duration of follow up in the study ranged from 0 to 48 months. The average BMI was $20.9(8.88) \mathrm{kg} / \mathrm{m}^{2}$. The most common comorbidities were HTN (81.4\%), followed by ambulatory dysfunction (46.7\%), DM (32.3\%), CKD (25.1\%), dementia (21.6\%), CAD (18.6\%), CHF (17.4\%), CVA (17.4\%), and Parkinson's disease (1.8\%).

\section{Etiology of Rhabdomyolysis}

Falls (with or without immobilization) was the most frequent cause of rhabdomyolysis in $56.9 \%$, followed by unknown etiology in $12 \%$, seizures in $5.4 \%$, immobilization in $5.4 \%$, sepsis in $4.8 \%$, statins in $2.4 \%$, trauma in $1.8 \%$, post surgery in $1.8 \%$, medication induced in $1.8 \%$, DKA in $1.2 \%$, neuroleptic malignant syndrome in $1.2 \%$, alcohol intoxication in $1.2 \%$, vigorous exercise in $0.6 \%$, post cardiac arrest $0.6 \%$, infection $0.6 \%$, and limb ischemia in $0.6 \%$.

\section{Medications and Laboratory Parameters}

The mean number of medications on admission was 7.52, with the range of $0-21$. The mean baseline creatinine, creatinine at diagnosis, and peak creatinine was 1.255 , 2.562 , and $2.876 \mathrm{mg} / \mathrm{dL}$ respectively. The mean baseline GFR, GFR at diagnosis, and peak decline in GFR were 76.94, 48.96, and $54.41 \mathrm{cc} / \mathrm{min}$ respectively. The mean CK at diagnosis and peak CK were 5097.22 and 6320.07. 
Table 1. Clinical characteristics in rhabdomyolysis elderly patients between AKI and non-AKI groups.

\begin{tabular}{|c|c|c|c|}
\hline Characteristics & Non-AKI $(n=50)$ & AKI (n=115) & $P$ values \\
\hline Age & $81.36(9.41)$ & $79.57(9.05)$ & $0.251^{*}$ \\
\hline Duration of follow up (months) & $13.21(13.83)$ & $12.26(12.99)$ & $0.679 *$ \\
\hline Body mass index & $21.94(8.89)$ & 20.43(9.19) & $0.728 *$ \\
\hline Number of medications pre-admission & $6.98(4.48)$ & $7.75(4.71)$ & $0.336^{*}$ \\
\hline Cr baseline & $1.33(1.46)$ & $1.22(0.62)$ & $0.631 *$ \\
\hline GFR baseline & $83.6(39.80)$ & $73.72(27.53)$ & $0.085^{*}$ \\
\hline $\mathrm{CK}$ at diagnosis & $3984.94(6652.55)$ & $5643.71(6608.37)$ & $0.146^{*}$ \\
\hline $\mathrm{Cr}$ at diagnosis & $1.68(2.69)$ & $2.90(2.91)$ & $0.012 *$ \\
\hline GFR at diagnosis & $75.60(37.68)$ & $37.72(23.24)$ & $<0.001 *$ \\
\hline CK peak & $5837.22(10171.41)$ & $6612.12(7155.41)$ & $0.578^{*}$ \\
\hline Potassium on admission & $4.32(0.80)$ & $4.47(1.01)$ & $0.363^{*}$ \\
\hline Phosphorus on admission & $3.65(1.36)$ & $4.37(1.22)$ & $0.034 *$ \\
\hline Albumin on admission & $3.01(0.76)$ & $2.67(0.72)$ & $0.025^{*}$ \\
\hline Cr peak & $1.72(2.69)$ & $3.34(3.19)$ & $0.001 *$ \\
\hline Peak decline in GFR & $81.72(43.99)$ & $42.55(26.95)$ & $<0.001 *$ \\
\hline CK discharge & $1443.13(3367.84)$ & $1531.25(4309.98)$ & $0.900 *$ \\
\hline Cr discharge & $1.23(1.25)$ & $1.75(1.97)$ & $0.091 *$ \\
\hline \multicolumn{4}{|l|}{ Race } \\
\hline African American & $37(74 \%)$ & $86(74.78 \%)$ & $0.582 * *$ \\
\hline Caucasian & $10(20 \%)$ & $22(19.13 \%)$ & \\
\hline Hispanic & $1(2 \%)$ & $1(0.87 \%)$ & \\
\hline Asian & $2(4 \%)$ & $2(1.73 \%)$ & \\
\hline Other & $0(0 \%)$ & $4(3.47 \%)$ & \\
\hline \multicolumn{4}{|l|}{ Sex } \\
\hline Male & $20(40 \%)$ & $58(50.43 \%)$ & $0.217 * *$ \\
\hline Female & $30(60 \%)$ & $57(49.56 \%)$ & \\
\hline Death in the same admission & $1(2 \%)$ & $15(13.04 \%)$ & $0.030 * *$ \\
\hline HTN & $39(78 \%)$ & $96(83.48 \%)$ & $0.337 * *$ \\
\hline $\mathrm{DM}$ & $12(24 \%)$ & $42(36.52 \%)$ & $0.107 * *$ \\
\hline $\mathrm{CHF}$ & $7(14 \%)$ & $22(19.13 \%)$ & $0.426 * *$ \\
\hline CAD & $6(12 \%)$ & $25(21.74 \%)$ & $0.141 * *$ \\
\hline Parkinson's disease & $0(0 \%)$ & $3(2.61 \%)$ & $0.249 * *$ \\
\hline Dementia & $11(22 \%)$ & $25(21.74 \%)$ & $0.970 * *$ \\
\hline Ambulatory dysfunction & $21(42 \%)$ & $56(48.69 \%)$ & $0.428 * *$ \\
\hline CVA & $5(10 \%)$ & $24(20.87 \%)$ & $0.092 * *$ \\
\hline CKD & $9(18 \%)$ & $33(28.70 \%)$ & $0.287 * *$ \\
\hline Cause of rhabdomyolysis & & & $0.381 * *$ \\
\hline Fall and/or immobilization & $38(76 \%)$ & $64(55.65 \%)$ & \\
\hline Statin induced & $0(0 \%)$ & $2(1.73 \%)$ & \\
\hline Accident/Trauma & $1(2 \%)$ & $2(1.73 \%)$ & \\
\hline Unknkown & $4(8 \%)$ & $16(13.91 \%)$ & \\
\hline Medication induced & $0(0 \%)$ & $3(2.61 \%)$ & \\
\hline Surgery & $1(2 \%)$ & $2(1.73 \%)$ & \\
\hline Burn & $0(0 \%)$ & $1(0.87 \%)$ & \\
\hline Seizure & $2(4 \%)$ & $7(6.09 \%)$ & \\
\hline Sepsis & $0(0 \%)$ & $8(6.95 \%)$ & \\
\hline Diabetes ketoacidosis & $0(0 \%)$ & $2(1.73 \%)$ & \\
\hline Neuroleptic Malignant Syndrome & $1(2 \%)$ & $1(0.87 \%)$ & \\
\hline Vigorous exercise & $1(2 \%)$ & $0(0 \%)$ & \\
\hline Alcohol & $1(2 \%)$ & $1(0.87 \%)$ & \\
\hline Post cardiac arrest & $0(0 \%)$ & $1(0.87 \%)$ & \\
\hline Lower extremity ischemia & $1(2 \%)$ & $0(0 \%)$ & \\
\hline Infection & $0(0 \%)$ & $1(0.87 \%)$ & \\
\hline Fall and statin induced & $0(0 \%)$ & $2(1.73 \%)$ & \\
\hline - & $24(48 \%)$ & $46(0.40 \%)$ & $0.339 * *$ \\
\hline
\end{tabular}

*Independent t-test **Chi-square test Cr-Creatinine, GFR-glomerular filtration rate, CK-creatnine kinase, HTN-hypertension, DMdiabetes mellitus, CHF-congestive heart failure, CAD-coronary artery disease, CVA-cerebrovascular accident, CKD-chronic kidney disease 
Table 2. Univariate analysis using Cox proportional hazard regression between overall survival and rhabdomyolysis variables.

\begin{tabular}{|c|c|c|c|}
\hline Characteristics & Hazard Ratio & 95\% Confident interval & P value \\
\hline Age & 1.016 & $0.984-1.050$ & 0.325 \\
\hline Sex & 1.3 .543694 & $0.900-3.190$ & 0.102 \\
\hline BMI & 1.016 & $0.737-1.400$ & 0.925 \\
\hline Hypertension & 0.454 & $0.199-1.031$ & 0.590 \\
\hline Diabetes & 1.216 & $0.577-2.561$ & 0.607 \\
\hline Congestive heart failure & 1.681 & $0.690-4.097$ & 0.253 \\
\hline Coronary artery disease & 0.954 & $0.408-2.230$ & 0.913 \\
\hline Parkinson's disease & 3.543 & $0.431-29.127$ & 0.239 \\
\hline Dementia & 1.761 & $0.843-3.680$ & 0.133 \\
\hline Ambulatory dysfunction & 0.426 & $0.203-0.895$ & 0.024 \\
\hline Cerebrovascular accident & 1.580 & $0.678-3.682$ & 0.289 \\
\hline Chronic kidney disease & 1.620 & $0.825-3.180$ & 0.161 \\
\hline Number of medications & 0.947 & $0.882-1.017$ & 0.135 \\
\hline Baseline Creatinine & 0.948 & $0.075-11.980$ & 0.967 \\
\hline Baseline GFR & 0.960 & $0.896-1.028$ & 0.247 \\
\hline $\mathrm{CK}$ at diagnosis & 1.000 & $0.999-1.000$ & 0.757 \\
\hline Creatinine at diagnosis & 0.276 & $0.022-3.450$ & 0.318 \\
\hline GFR at diagnosis & 0.928 & $0.822-1.048$ & 0.227 \\
\hline Peak CK & 1.000 & $1.000-1.001$ & 0.506 \\
\hline Serum Potassium at diagnosis & 1.025 & $0.377-2.792$ & 0.961 \\
\hline $\begin{array}{l}\text { Serum Phosphorus at } \\
\text { diagnosis }\end{array}$ & 0.978 & $0.479-1.996$ & 0.951 \\
\hline Serum troponin at diagnosis & 1.000 & $0.999-1.000$ & 0.287 \\
\hline Serum albumin at diagnosis & 1.268 & $0.460-3.495$ & 0.646 \\
\hline Peak creatinine & 3.200 & $0.340-30.091$ & 0.309 \\
\hline Peak GFR & 1.096 & $0.960-1.253$ & 0.176 \\
\hline CK at discharge & 1.001 & $0.999-1.002$ & 0.265 \\
\hline Creatinine at discharge & 0.963 & $0.191-4.862$ & 0.964 \\
\hline Acute kidney injury (Yes/No) & 3.043 & $1.90-7.781$ & 0.020 \\
\hline $\begin{array}{l}\text { Need for dialysis } \\
\text { (Yes/No/ESRD) }\end{array}$ & 2.013 & $0.884-4.582$ & 0.096 \\
\hline
\end{tabular}

\section{AKI and Rhabdomyolysis}

The incidence of AKI was $68.9 \%$. We divided the patients into two groups according to the presence or absence of AKI during hospitalization. Significant associations were found between AKI and higher phosphorus on admission (4.37 vs $3.65, P 0.034)$, lower albumin on admission $(2.67$ vs 3.01, P0.025), and higher percentage of death during the same admission (13.04 vs $2.00 \%, \mathrm{P} 0.030$ ) (Table 1). In addition, it was expected that AKI group had higher $\mathrm{Cr}$ at diagnosis (2.90 vs $1.68, P=0.012$ ), lower GFR at diagnosis (37.72 vs $75.60, P<0.001$ ), higher $\mathrm{Cr}$ peak (3.34 vs $1.72, P=0.001)$, and lower peak decline in GFR (42.55 vs $81.72, P<0.001)$. There was no statistical difference between $\mathrm{Cr}$ at discharge or $\mathrm{CK}$ at discharge between two groups.

\section{Correlation between peak $C K$ and peak $C r$ or peak decline in GFR}

We found neither statistically significant correlation between peak CK and peak Cr (Pearson's correlation coefficient 0.019, $P=0.805$ ), nor peak $\mathrm{CK}$ and peak decline in GFR (Pearson's correlation coefficient 0.037, $P=0.638)$.

\section{Prognostic factors for overall survival}

There were 45 deaths $(21 \%)$ over the span of 4 years. All cause mortality during the same hospitalization was $10.17 \%$. The median survival of this study was 7 months.

Univariate analysis using Cox proportional hazard regression between overall survival and each variable was analyzed (Table 2). We allowed all variables with a univariate $p$-value less than 0.25 to enter into a 
multivariate analysis. Once in a multivariate analysis, a backwards selection procedure was used to remove variables with a $\mathrm{p}$-value greater than 0.05 . This generated three independent predictors of overall survival in this population: number of pre-admission medications ( $<8$ vs 28) (Hazard Ratio (HR) 2.391, 95\% Confident interval
(CI) $1.166-4.902$, GFR (<50 vs $\geq 50)(\mathrm{HR} 2.039,95 \% \mathrm{CI}$ 1.004-4.141), and AKI (HR 3.326, 95\% CI 1.139-9.715). Therefore, we found that Med No., AKI, and GFR are independent predictors for overall survival for rhabdomyolysis in elderly patients (Table 3 ).

Table 3. Multivariate analysis using backward selection model

\begin{tabular}{llll}
\hline Characteristics & Hazard Ratio & 95\% Confident interval & $\boldsymbol{P}$ value \\
\hline Number of Medications $(<8 \mathrm{vs} \geq 8)$ & 2.391 & $1.166-4.902$ & 0.017 \\
Peak GFR $(<30$ vs $\geq 30 \mathrm{mg} / \mathrm{dL})$ & 2.039 & $1.004-4.141$ & 0.049 \\
Acute kidney injury & 3.326 & $1.139-9.715$ & 0.028 \\
\hline
\end{tabular}

\section{DISCUSSION}

In this study, we described for the first time the epidemiology and prognosis of rhabdomyolysis in the elderly. Falls (with or without immobilization) was the most frequent cause of rhabdomyolysis in the elderly in $56.9 \%$, compared with previous studies in adult patients which revealed illicit drug use and alcohol [14-16, 21], infectious [15, 18], ischemic [17], and seizure [13] as the most common etiology of rhabdomyolysis.

Approximately $34 \%$ of elderly age above 65 years old experience at least one fall per year [22]. Fractures, decrease in functional status, and risk for institutionalization are known consequences of falls. Besides the physical consequences, falls can also lead to mental consequences, such as, fear of fall, depression, social isolation, and lack of confidence [23]. Further studies about fall prevention strategies would definitely be beneficial in terms of preventing rhabdomyolysis, fractures, deconditioning in the elderly and other mental consequences of falls in the elderly [24].

Polypharmarcy, the overuse of prescription medications more than medically necessary, is highly prevalent in elderly patients [25]. It leads to multiple negative consequences, including, falls, functional decline, cognitive impairments, and malnutrition states [25]. In this study, we found that average number of medications was 7.52 , with the range of $0-21$. More importantly, we discovered that the number of medications pre-admission $(>8)$ was associated with shorter overall survival in elderly patients with rhabdomyolysis.

It has been approximated that AKI secondary to rhabdomyolysis is responsible for $7-10 \%$ of all cause AKI in the United States [10]. The incidence of AKI was $68.9 \%$ in our study. This incidence is higher compared to the previous study by Melli et al [21]. and Veenstra et al [17], who reported an incidence of rhabdomyolysis $46 \%$ and $51 \%$ respectively. One possible explanation of our higher incidence of AKI is the age difference. Our study included elderly patients age above 65 years old, compared with both previous studies, which included all adults and children age from 4 years [21] and 16 years [17]. There are known associations between AKI and aging, for example, older adults in critical care settings had $20 \%$ higher rate of developing AKI compared to younger adults [26].

We performed further analysis by categorizing patients into AKI and non-AKI group. We found that patients with AKI had significantly higher phosphorus levels and lower albumin levels on admission. These findings were consistent with the study by ward et al. who studied the profile of AKI in rhabdomyolysis in 157 participants. They found higher serum phosphorus level and lower serum albumin level in renal failure group compared with non-renal failure group (1.85 vs 1.06 $\mathrm{mmol} / \mathrm{L}, \mathrm{P}<0.001$ and 30.8 vs $35.9 \mathrm{~g} / \mathrm{L}, \mathrm{P}=0.010$ ) [27]. In addition, there were no significant differences between $\mathrm{CK}$ and $\mathrm{Cr}$ levels upon discharge between groups.

Statins responsible for approximately $2.4 \%$ cases of rhabdomyolysis in our study. The incidence is slightly lower than the $4.2 \%$ rate reported by Melli et al [21]. There are several well-established studies about statinassociated adverse muscular events. In 2015, Iwere et al. conducted a systematic review and meta-analysis about statins and myopathy in elderly patients[28]. They included 8 studies and found that statins increase the risk of rhabdomyolysis in the elderly with the odd ratio of 2.93 (95\% CI 0.30-28.18). Based on our results, previous study by Melli et al [21], and the above-mentioned metaanalysis, statins increase the risk of rhabdomyolysis in the elderly compared to general population, however, the event is generally uncommon.

There are some limitations in our study. The first limitation is in the study's nature. Due to the fact that this study is a retrospective chart review study, some data may 
be underestimated or underreported, such as, comorbidities and etiology of rhabdomyolysis. The second limitation is disparities in terms of population group. The study population was predominantly African American (73.7\%) thus limiting extrapolation of this data across all populations. Lastly, we have limited sample size of 165 patients. Larger prospective studies in elderly patients would be crucial in elucidating the relationship between falls, geriatric syndromes, and rhabdomyolysis in the elderly.

\section{Conclusions}

To our knowledge, this is the first epidemiological study of rhabdomyolysis in the elderly. We demonstrated that falls (with and without immobilization) were the most common etiologies for rhabdomyolysis in the elderly. Approximately two-third of patients developed AKI. The number of medications pre-admission $(>8)$, peak decline in GFR $(<30 \mathrm{cc} / \mathrm{min})$, and the presence of AKI are associated with shorter overall survival and can serve as potential independent prognostic markers for rhabdomyolysis in elderly patients.

\section{Acknowledgements}

SW, CK, and PP conceived of the study and together with JR and AR, collaborated on the design of the study. SW, $\mathrm{CK}$, and PP carried out the data collection. SW performed the data analysis and drafted the manuscript. All authors contributed substantially and approved the final manuscript. The authors declare that there is no conflict of interest. There is no funding source for this research.

\section{Reference}

[1] Chavez LO, Leon M, Einav S, Varon J (2016). Beyond muscle destruction: a systematic review of rhabdomyolysis for clinical practice. Crit Care, 20: 135

[2] Huerta-Alardin AL, Varon J, Marik PE (2005). Benchto-bedside review: Rhabdomyolysis -- an overview for clinicians. Crit Care, 9: 158-169

[3] Keltz E, Khan FY, Mann G (2013). Rhabdomyolysis. The role of diagnostic and prognostic factors. Muscles Ligaments Tendons J, 3: 303-312

[4] Scalco RS, Gardiner AR, Pitceathly RD, Zanoteli E, Becker J, Holton JL, et al. (2015). Rhabdomyolysis: a genetic perspective. Orphanet J Rare Dis, 10: 51

[5] Kim KK (1996). Exogenous causes of myoglobinuria-review of 26 cases. J Korean Med Sci, 11: 342-346

[6] Singh U, Scheld WM (1996). Infectious etiologies of rhabdomyolysis: three case reports and review. Clin Infect Dis, 22: 642-649

[7] Byrd RP, Jr., Roy TM (1998). Rhabdomyolysis and bacterial pneumonia. Respir Med, 92: 359-362
Khan FY (2009). Rhabdomyolysis: a review of the literature. Neth J Med, 67: 272-283

Torres PA, Helmstetter JA, Kaye AM, Kaye AD (2015). Rhabdomyolysis: pathogenesis, diagnosis, and treatment. Ochsner J, 15: 58-69

Bosch X, Poch E, Grau JM (2009). Rhabdomyolysis and acute kidney injury. N Engl J Med, 361: 62-72

Criddle LM (2003). Rhabdomyolysis. Pathophysiology, recognition, and management. Crit Care Nurse, 23: 14-22, 24-16, 28 passim; quiz 31-12 Lima RS, da Silva Junior GB, Liborio AB, Daher Ede F (2008). Acute kidney injury due to rhabdomyolysis. Saudi J Kidney Dis Transpl, 19: 721-729

Grossman RA, Hamilton RW, Morse BM, Penn AS, Goldberg M (1974). Nontraumatic rhabdomyolysis and acute renal failure. N Engl J Med, 291: 807-811

Koffler A, Friedler RM, Massry SG (1976). Acute renal failure due to nontraumatic rhabdomyolysis. Ann Intern Med, 85: 23-28

Eneas JF, Schoenfeld PY, Humphreys MH (1979). The effect of infusion of mannitol-sodium bicarbonate on the clinical course of myoglobinuria. Arch Intern Med, 139: 801-805

Gabow PA, Kaehny WD, Kelleher SP (1982). The spectrum of rhabdomyolysis. Medicine (Baltimore), 61: $141-152$

Veenstra J, Smit WM, Krediet RT, Arisz L (1994). Relationship between elevated creatine phosphokinase and the clinical spectrum of rhabdomyolysis. Nephrol Dial Transplant, 9: 637-641

Blanco JR, Zabalza M, Salcedo J, Echeverria L, Garcia A, Vallejo M (2002). Rhabdomyolysis of infectious and noninfectious causes. South Med J, 95: $542-544$

McMahon GM, Zeng X, Waikar SS (2013). A risk prediction score for kidney failure or mortality in rhabdomyolysis. JAMA Intern Med, 173: 1821-1828 Nance JR, Mammen AL (2015). Diagnostic evaluation of rhabdomyolysis. Muscle Nerve, 51: 793-810

Melli G, Chaudhry V, Cornblath DR (2005). Rhabdomyolysis: an evaluation of 475 hospitalized patients. Medicine (Baltimore), 84: 377-385

Rubenstein LZ, Josephson KR (2002). The epidemiology of falls and syncope. Clin Geriatr Med, 18: $141-158$

Luk JK, Chan TY, Chan DK (2015). Falls prevention in the elderly: translating evidence into practice. Hong Kong Med J, 21: 165-171

Ungar A, Rafanelli M, Iacomelli I, Brunetti MA, Ceccofiglio A, Tesi F, et al. (2013). Fall prevention in the elderly. Clin Cases Miner Bone Metab, 10: 91-95 Maher RL, Hanlon J, Hajjar ER (2014). Clinical consequences of polypharmacy in elderly. Expert Opin Drug Saf, 13: 57-65

Hain D, Paixao R (2015). The perfect storm: older adults and acute kidney injury. Crit Care Nurs Q, 38: 271-279

Ward MM (1988). Factors predictive of acute renal failure in rhabdomyolysis. Arch Intern Med, 148: $1553-1557$ 
[28] Iwere RB, Hewitt J (2015). Myopathy in older people receiving statin therapy: a systematic review and metaanalysis. Br J Clin Pharmacol, 80: 363-371 